\title{
Squeeze the lemon: balancing as a way to use every drop of energy in a Lithium-ion battery
}

\author{
Federico Baronti, Roberto Roncella, Roberto Saletti \\ Dipartimento Ingegneria dell'Informazione, University of Pisa \\ Via G.Caruso 16, 56126 PISA, Italy
}

\begin{abstract}
This work discusses recent research results obtained in tackling one of the most limiting factor for an effective use of a Lithium-ion battery: the charge unbalance between the cells constituting the battery. First, it is recalled how unbalancing affects the performance of a battery consisting of series-connected cells, then some possible techniques to balance the battery are described and compared to each other. The comparison is made by modeling the balancing circuit topologies and by performing statistical simulations. Finally, we describe two balancing circuits that efficiently address the problem and we report on the experimental results that validate the circuits.
\end{abstract}

\section{Introduction}

Lithium-ion battery technology has determined the explosion of the portable electronic device market (laptop, personal digital assistant, smart-phones), as it provides very high energy and power densities if compared to previously adopted technologies [1]. The introduction of Lithium-ion batteries has made it viable applications that seemed inconceivable before. The Electric Vehicle (EV) automotive market also seems to have migrated from Lead-acid or NiMH batteries to the more performing Lithium-ion batteries as new EVs are now equipped with the latter. Therefore, Lithium-ion is becoming the most widely adopted technology for rechargeable energy storage systems, even in medium- high-power applications. Unfortunately, Lithium-ion batteries are very fragile as they are sensitive to the operating conditions. Permanent damages and even flames may occur if the battery is overcharged, undercharged or operated outside the safe temperature range. Thus, Lithium-ion batteries are always provided with an electronic control system that is in charge of monitoring, managing and preserving the safety of the battery. This circuit is generally called Battery Management System (BMS). A BMS is usually interfaced to upper level control systems in the considered application to provide valuable information such as the State of Charge $(\mathrm{SoC})$ and State of Health $(\mathrm{SoH})$ of the battery cells [2]. Besides some accidents reported on vehicles or planes and the issues that research efforts are trying to solve, the growth of the Lithium-ion battery market continues. One of the problems not efficiently solved yet for an effective use of a Lithium-ion battery is the charge unbalance between the series-connected cells building up the 
battery. As the Lithium-ion battery technology provides elementary cells with voltages of the order of $3 \div 4 \mathrm{~V}$, many cells are arranged in series to reach the voltage level required by the application, even hundreds of Volt in EVs. Possible mismatches in the physical properties or in the operating conditions of the cells determine a charge imbalance that makes it impossible to utilize all the energy in the battery without exceeding the safety limits of the cells [3]. Thus, the BMS must provide the battery pack with a balancing function also. This paper reviews and compares by means of statistical simulations some of the most popular balancing techniques. The implementations of two different balancing topologies are finally described, together with the experimental results measured on the related balancing circuits.

\section{Battery balancing circuit topologies}

Cell unbalance is a major issue in a Li-ion battery consisting of series-connected cells, because battery recharge must be stopped when one cell reaches the charge cut-off voltage, even if the other cells are not completely charged. On the other hand, the discharge is interrupted when the least charged cell reaches the discharge cut-off voltage before the others, even if there is still some residual energy stored in the battery. The basic idea behind balancing is to bring all the unbalanced cells to the same charge level at the end of the balancing process. In this way, all the cells (assuming that they have the same capacity) will reach the charge/discharge cut-off voltage limits simultaneously, thus allowing the use of every drop of energy than can be stored in the battery.

Thus, balancing requires that every cell must be individually accessed to modify its charge level. The easiest way to balance a battery is to dissipate the extra energy in a resistor placed in parallel to the cell until all the cells reach the same charge level. This is called passive balancing. Instead of dissipating energy, active balancing aims at saving energy by transferring charges from the most charged cells to the least charged ones. Several topologies are possible, according to the way by which charges are transferred between the cells $[4,5]$. In this work, we define the Cell to Cell, Cell to Pack and Pack to Cell topologies if the charges are exchanged between two individual cells, from one particular cell to the entire battery pack, or from the entire battery pack to one particular cell, respectively. These active balancing methodologies are based on a matrix of switches by which each cell of the battery is individually accessed to modify its charge. Each topology can be modeled with a very simple model of charge transfer, in which the charges are moved with energy efficiency $\eta$ by means of a bidirectional DC/DC converter with $N+1$ ports [6], connected to the $N$ cells and to the battery pack terminals. The general model of the balancing network is shown in Fig. 1.

As detailed in [6], the optimum sequence of charge transfers can be found for each topology that makes the battery balanced. Unfortunately, the charge transfers occur with a certain energy efficiency as every transfer dissipates energy. The optimum sequence comes from the minimization of the charges to be 


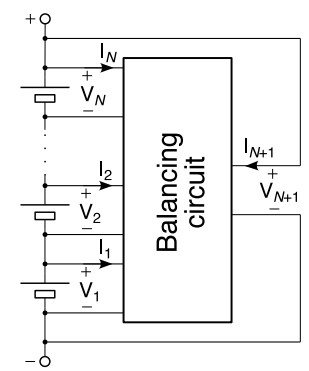

Fig. 1. General model of a balancing network based on a DC/DC converter and $N+1$ ports [6].

transferred during balancing. This means that every unbalanced cell must reach the final charge level, which is identical for all the cells, in a monotonic way, i.e., always donating or accepting charges only.

A comparison among the different balancing topologies in terms of balancing time and energy loss during balancing is carried out in [6], by applying the above described model and strategy to different distributions, statistically chosen, of unbalance states of the battery cells. The following assumptions are made. $Q_{\max }$ is the maximum charge stored in any cell. The maximum $\mathrm{SoC}$ mismatch considered in the example is $10 \%$, as the most charged cell is at $Q_{\max }$, while the least charged cells is at $0.9 \cdot Q_{\max }$. The other cells are charged at $Q_{h}$, with $0.9 \cdot Q_{\max }<Q_{h}<Q_{\max }, h \in 1 \ldots N-2$ and $Q_{h}$ randomly distributed with a uniform distribution between the two limit values. This means that we find an unbalanced battery with $10 \%$ maximum $S o C$ mismatch, with all the cells between $90 \%$ and $100 \%$ of the maximum charge, in every statistical experiment. The optimum balancing algorithm is then applied and the values of the balancing time and the energy losses are calculated. Two comparison parameters, the balancing time figure $F_{\text {time }}$ and the energy loss figure $F_{\text {loss }}$, are defined as the ratio between the balancing time and the energy loss, respectively, divided by the relevant values calculated for the passive balancing. Values of $F_{\text {time }}$ and $F_{\text {loss }}$ less than 1 mean that the active balancing techniques have performed better than the passive one. The experiment is repeated with 100,000 different unbalance configurations with the following additional parameters: the current $I_{\mathrm{sh}}$ in the shunt resistor of passive balancing is $200 \mathrm{~mA}$, the DC/DC balancing current $I_{\text {bal }}$ is $1 \mathrm{~A}$ and the converter energy efficiency $\eta$ is 0.85 . The probability density function (PDF) of the random variables $F_{\text {time }}$ and $F_{\text {loss }}$ are finally calculated. Fig. 2 shows the results.

The diagrams show that active balancing performs better or equal to passive balancing in time, and by far better if energy losses are considered. However, the simulation results show that there are significant differences between the various active topologies. In particular, the Cell to Cell configuration clearly outperforms the other methods in terms of both balancing time and energy loss. 

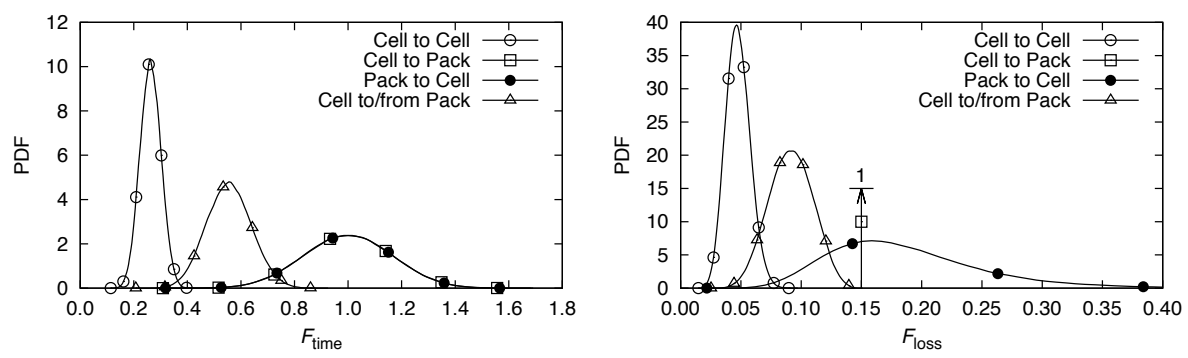

Fig. 2. Probability Density Function $(\mathrm{PDF})$ of the balancing time figure $F_{\text {time }}$ (left) and the balancing energy loss figure $F_{\text {time }}$ (right) [6].

The energy loss comparison is also performed by varying the DC/DC converter efficiency $\eta$, as it is shown in Fig 3, where the mean value of the energy loss figure $F_{\text {loss }}$ is reported as a function of $\eta$. It is amazing to see that active balancing may be worse than passive balancing (a value greater than 1) if the DC/DC converter is of low quality and its efficiency drops below $50 \%$, for the Pack to Cell configuration, one of the most popular topologies.

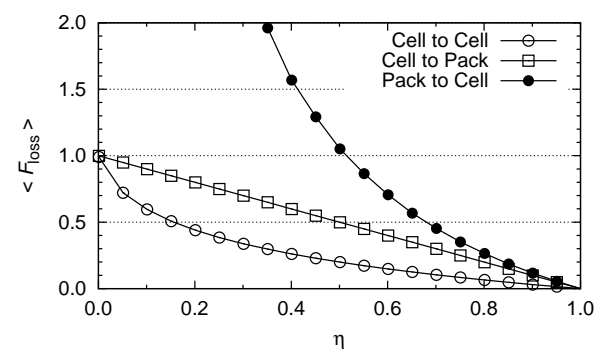

Fig. 3. Mean value $<F_{\text {loss }}>$ of $F_{\text {loss }}$ as a function of the converter efficiency $\eta$ [6].

In conclusion, the study shows that, besides the converter efficiency, the most important factor to efficiently achieve battery balancing and thus squeeze all the energy stored in a battery is the topology used to transfer charges between the battery cells.

\section{Balancing circuit implementations}

Two balancing circuits that implement the Cell to Cell and Pack to Cell balancing techniques are described hereafter. 


\subsection{Cell-to-Cell balancing circuit}

The first circuit is shown on the left side of Fig. 4. The cells are connected by means of a switch matrix consisting of MOS transistors. The DC/DC converter is bidirectional and connects the cell selected by the switch matrix with a supercapacitor (SCAP) that acts as a temporary energy storage tank. The idea applied in this circuit is to extract energy from one cell, store it in the SCAP and then return it back to another properly selected cell. At the end of the two-way transfer we have succeeded in moving charges from one cell to another, thus applying the cell-to-cell balancing topology. The circuit was applied to a battery consisting of $1140 \mathrm{Ah}$ cells with Nickel Manganese Cobalt (NMC) cathode. Details on the circuit implementation and the experimental validation can be found in [7].
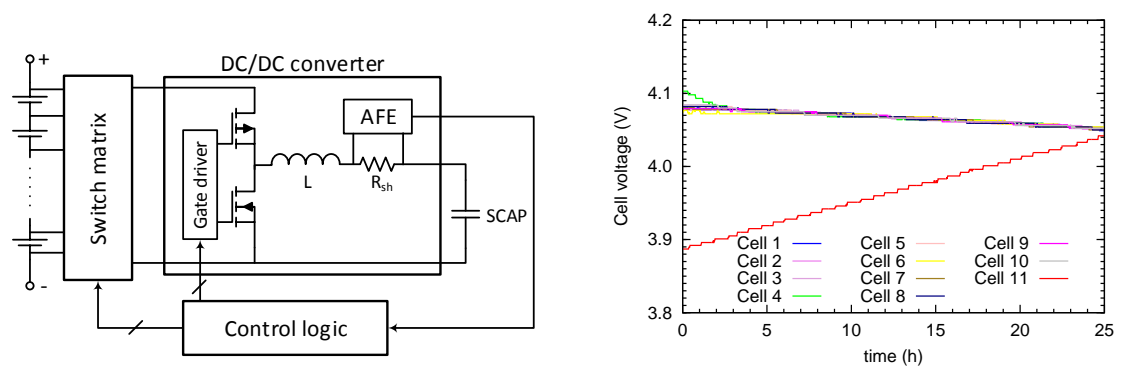

Fig. 4. Schematic circuit of the balancing circuit implementing the Cell-to-Cell topology (left). Cell voltages as a function of time, showing that balancing is finally achieved after starting with a large unbalanced configuration (right) [7].

The right side of Fig. 4 shows the voltages of the battery cells as a function of time during a balancing experiment. The battery is strongly unbalanced, as one cell (Cell 11) shows a SoC almost $20 \%$ less than the other cells. It is worth noting how balancing is recovered by moving from the other cells the charges needed to equalize the battery. The balancing procedure lasts around $25 \mathrm{~h}$ and is very efficient as it only costs $1 \%$ of the battery energy. In fact, the measured efficiency of the energy transfer is well over $75 \%$. Finally, an energy saving of a factor larger than 6 is obtained if compared to the passive balancing technique applied to the same unbalanced condition.

\subsection{Pack-to-Cell balancing circuit}

The circuit implementing the Pack-to-cell balancing topology is shown in Fig. 5 and is described in details in [8] and [9]. The circuit consists of a DC/DC converter fed by the entire battery voltage that provides charges to one particular cell selected by the switch matrix. A peculiar feature of this circuit is the presence of an additional switch that enables the balancing between different battery 
modules connected with a circular balancing bus. The circuit performs the balancing of 460 Ah Lithium-ion with Lithium Iron Phosphate (LFP) cathode cells with balancing currents of $1.5 \mathrm{~A}$. The switch matrix is realized with MOS switches characterized by an on-resistance of about $7.5 \mathrm{~m} \Omega$, thus leading to a rather low dissipation on them. The measured balancing efficiency is around $70 \%$.

Fig. 5 also shows the $S o C$ of the battery cells before and after a balancing process that starts with cell 3 unbalanced of $10 \%$. It is worth noting how a balanced configuration of the battery is restored, allowing the exploitation of the full capacity of the battery. The cell voltages are also reported to show the voltage relaxation phenomena occurring after the balancing end.
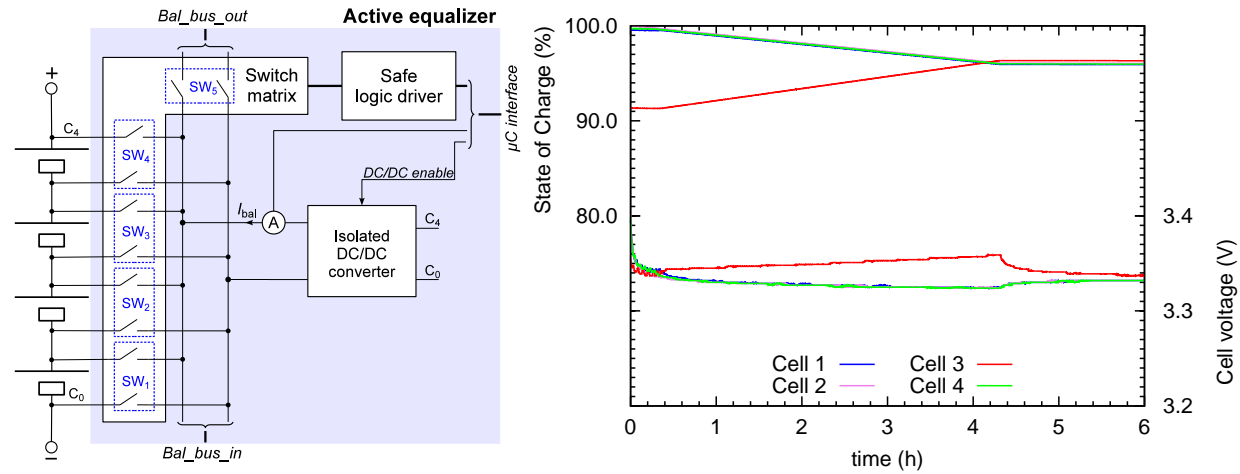

Fig. 5. Schematic circuit of the balancing circuit implementing the Pack-to-Cell topology (left). State of Charge and voltage of the cells as a function of time, showing that balancing is restored (right) [9].

\section{Conclusions}

The paper presents a survey of research efforts aimed to achieve the efficient balancing of a Lithium-ion battery. Balancing a battery allows the exploitation of every drop of energy stored in the battery, otherwise not fully usable. It is shown by statistical simulations that active balancing performed by transferring charges from the most charged cells to least charged ones is the most efficient technique for both balancing time and energy loss figures of merit. Two circuits that implement different balancing techniques are described and the results obtained during their experimental validation are finally presented.

\section{References}

1. Whittingham, M.S.: History, Evolution, and Future Status of Energy Storage. Proceedings of the IEEE 100(Special Centennial Issue) (May 2012) 1518-1534 
2. Lu, L., Han, X., Li, J., Hua, J., Ouyang, M.: A review on the key issues for lithiumion battery management in electric vehicles. Journal of Power Sources 226 (March 2013) 272-288

3. Zhong, L., Zhang, C., He, Y., Chen, Z.: A method for the estimation of the battery pack state of charge based on in-pack cells uniformity analysis. Applied Energy $\mathbf{1 1 3 ( 0 )}$ (January 2014) 558-564

4. Gallardo-Lozano, J., Romero-Cadaval, E., Milanes-Montero, M.I., GuerreroMartinez, M.A.: Battery equalization active methods. Journal of Power Sources 246 (January 2014) 934-949

5. Daowd, M., Omar, N., Van Den Bossche, P., Van Mierlo, J.: Passive and active battery balancing comparison based on MATLAB simulation. In: 2011 IEEE Vehicle Power and Propulsion Conference, IEEE (September 2011) 1-7

6. Baronti, F., Roncella, R., Saletti, R.: Performance comparison of active balancing techniques for lithium-ion batteries. Journal of Power Sources 267 (December 2014) 603-609

7. Baronti, F., Roncella, R., Saletti, R., Zamboni, W.: Experimental Validation of an Efficient Charge Equalization System for Lithium-Ion Batteries. In: 2014 23rd IEEE International Symposium on Industrial Electronics (ISIE), Istambul (2014) 1-6

8. Baronti, F., Fantechi, G., Roncella, R., Saletti, R.: High-Efficiency Digitally Controlled Charge Equalizer for Series-Connected Cells Based on Switching Converter and Super-Capacitor. IEEE Transactions on Industrial Informatics 9(2) (May 2013) 1139-1147

9. Baronti, F., Fantechi, G., Roncella, R., Saletti, R., Pede, G., Vellucci, F.: Design of the battery management system of LiFePO4 batteries for electric off-road vehicles. In: 2013 IEEE International Symposium on Industrial Electronics ISIE, Taipei, IEEE (May 2013) 1-6 